\title{
Laparoscopic Local Resection through Subserosal Dissection with Endoscopic Air-Insufflation for Submucosal Tumors Located Near the Esophagogastric Junction
}

\author{
In-Kyeong Kim, M.D. ${ }^{1}$, Ji-Ho Park, M.D. ${ }^{1}$, Young-Joon Lee, M.D., Ph.D. ${ }^{2}$, Sang-Ho Jeong, M.D., Ph.D. ${ }^{2}$, \\ Tae Han Kim, M.D., Ph.D. ${ }^{2}$, Dong-Hwan Kim, M.D. ${ }^{1}$, Han-Gil Kim, M.D. ${ }^{1}$, Jin-Kyu Cho, M.D. ${ }^{1}$, Jae-Myung Kim, M.D. ${ }^{1}$, \\ Seung-Jin Kwag, M.D., Ph.D. ${ }^{1}$, Ju-Yeon Kim, M.D., Ph.D. ${ }^{1}$, Chi-Young Jeong, M.D., Ph.D. ${ }^{1}$, Young-tae Ju, M.D., Ph.D. ${ }^{1}$, \\ Eun-Jung Jung, M.D., Ph.D. ${ }^{1}$, Soon-Chan Hong, M.D., Ph.D. ${ }^{1}$ \\ 'Department of Surgery, Gyeongsang National University Hospital, College of Medicine, Gyeongsang National University, Jinju, ${ }^{2}$ Department of Surgery, \\ Gyeongsang National University Changwon Hospital, College of Medicine, Gyeongsang National University, Changwon, Korea
}

Purpose: A novel resection method, namely, laparoscopic local resection through subserosal dissection with endoscopic air-insufflation (LRSDEA) was used for submucosal tumors located near the esophagogastric junction (SMT-EGJ) to avoid major gastric resection.

Methods: A total of 9 cases underwent LRSDEA. We sequentially performed: laparoscopic dissections around EGJ, subserosal dissections around SMTs using laparoscopic electrocautery and ultrasonic shears, and finally, enucleation of SMTs. During these procedures, intraoperative endoscopic tumor localization, as well as endoscopic air-insufflation allowed for safe resection. These procedures are shown in the supplementary video clip. The clinicopathological characteristics and surgical results were analyzed.

Results: All laparoscopic procedures were successfully performed without requiring a major gastrectomy. The mean operation time was 126.1 minutes, and estimated blood loss was $12.0 \mathrm{ml}$. There were no postoperative complications. Pathological diagnoses were 6 leiomyomas, 2 gastrointestinal stromal tumors, and 1 gastric duplication.

Conclusion: LRSDEA is an effective and safe treatment option for SMT-EGJ, as major resection of the stomach is avoided.

Keywords: Laparoscopy, Stomach neoplasm, Gastrointestinal stromal tumor, Esophagogastric junction, Endoscopy

This is an Open Access article distributed under the terms of the Creative Commons Attribution Non-Commercial License (http:/ creativecommons.org/licenses/by-nc/4.0/) which permits unrestricted non-commercial use, distribution, and reproduction in any medium, provided the original work is properly cited.
Received July 22, 2020

Revised October 26, 2020

Accepted November 10, 2020

Corresponding author

Young-Joon Lee

Department of Surgery, Gyeongsang National University Hospital, College of Medicine, Gyeongsang National University, 79 Gangnam-ro, Jinju 62727 , Korea

Tel: $+82-55-750-8615$

Fax: +82-55-757-5442

E-mail: yjleegnu@gmail.com

ORCID:

https://orcid.org/0000-0002-1735-3385

In-Kyeong Kim and Ji-Ho Park contributed equally to this study.

Copyright ( $\odot 2020$ The Journal of Minimally Invasive Surgery. All rights reserved.

\section{INTRODUCTION}

The main procedure for the treatment of a localized gastric submucosal tumor (SMT) is complete surgical resection. ${ }^{1}$ Laparoscopic gastric surgery is performed globally, and therefore, laparoscopic local resection is the main procedure for the treatment of various types of gastric SMTs, such as leiomyomas, gas- trointestinal stromal tumors (GISTs), and even malignant GISTs. ${ }^{2}$ These tumors rarely involve lymph node metastasis, and are thus suitable for laparoscopic local resection requiring excision with a negative resection margin. ${ }^{3}$ Moreover, the development of various laparoscopic stapling devices and surgical techniques has made laparoscopic wedge resection an attractive alternative to conventional gastrectomies. ${ }^{4}$ Major gastric resection, such as 
subtotal or total gastrectomy, is generally only performed if the tumor is giant, highly malignant, or if the functioning of the EGJ or the pylorus is compromised.

Currently, ablation of SMTs for which local resection is sufficient is performed by using various laparo -endoscopic surgical methods, including conventional wedge resection using a linear stapler, laparoscopic and endoscopic cooperative surgery (LECS), modified LECS, transgastric resection, intragastric resection, and endoscopic full-thickness resection. These methods have been reported for the resection of submucosal tumors located near the esophagogastric junction (SMT-EGJ), and have certain advantages with regard to the preservation of the gastric inlet. ${ }^{5-11}$ Nevertheless, SMT-EGJ still poses challenges for gastric surgeons due to the high risk of stenosis, deformity in the gastric inlet, and even leakage at the resected site. In particular, SMT-EGJ with endophytic growth patterns are difficult to localize using laparoscopy, as this would require relatively large resection of the normal gastric wall, and is often associated with postoperative complications such as reflux, dysphagia, nausea, and vomiting.

In this technical report we have introduced a safe and effective technique for the resection of endophytic SMT-EGJ. Laparoscopic local resection via subserosal dissection with endoscopic air-insufflation (LRSDEA) is a new surgical method which could avoid major resection of the stomach, and thus preserve the anatomical structure of the EGJ.

\section{MATERIALS AND METHODS}

\section{Patients and data collection}

Between 2014 and 2018, 9 patients with SMT-EGJ underwent LRSDEA in our medical center. All patients underwent a preoperative assessment, which included a history taking, physical examination, laboratory blood test, chest X-ray, electrocardiography, and pulmonary function test for general anesthesia. A preoperative esophago-gastroscopy, endoscopic ultrasound (EUS), and abdominal computed tomography (CT) scan were essential for all patients, whose results were reviewed and assessed by an experienced radiologist and gastroenterologist for the malignancy potential of the tumors.

Signs of gastric SMTs suggesting that resection was necessary were: a tumor larger than $2 \mathrm{~cm}$, an increase in tumor size through serial endoscopic surveillance, and the presence of symptoms associated SMTs. Tumors that are smaller than 2 $\mathrm{cm}$ and have high-risk features on endoscopy and EUS, such as irregular borders, ulceration, and heterogeneity, should be removed surgically. The National Comprehensive Cancer Network (NCCN) Task Force Report suggested that laparoscopic resection is a reasonably safe and feasible procedure. ${ }^{12}$ In the case of SMTEGJ, proximal gastrectomy was preferred for tumors of $5 \mathrm{~cm}$ or more, as well as those suspected of having a strong malignant potential during the preoperative examinations, particularly the esophagogastroduodenoscopy (EGD), EUS, and CT scan. SMTs exhibiting exophytic growth using diagnostic laparoscopy were resected using either conventional wedge resection, or the LECS method. In the case of endophytic dominant type SMTs, which were barely visible via laparoscopy outside the stomach, the tumors were removed using LRSDEA. Cases where the tumor center was located $2 \mathrm{~cm}$ above or $5 \mathrm{~cm}$ below the EGJ were defined as SMT-EGJ.

All data were reviewed retrospectively, including information on patient demographics, diagnostic workup, operative and pathological findings, postoperative hospital care, and follow-up surveillance.

The LRSDEA procedure was performed once consent was obtained, and the method was thoroughly explained. Approval was obtained from the Institutional Review Board before the initiation of this review (2019-10-036-003).

\section{Surgical procedure of LRSDEA}

Laparoscopic surgery was performed under general endotracheal anesthesia. The operator and laparoscopist stood on the right of the patient, and first assistant on the left. A $12 \mathrm{~mm}$ sized trocar was inserted into the infraumbilical region using the open method, and a carbon dioxide pneumoperitoneum was established at an intra-abdominal pressure of 10 12 $\mathrm{mmHg}$. Additionally, a $12 \mathrm{~mm}$ and two $5 \mathrm{~mm}$ sized trocars were inserted into the upper quadrants; the positions of which differ among the patients, depending on tumor location. The abdominal cavity was fully examined to identify any ascites or intraperitoneal metastases. The liver was retracted by a suture, which was passed through the mid pars condensa of the lesser omentum and the falciform ligament.

After laparoscopic dissection around the lesser curvature and abdominal EGJ area (Fig. 1A), the location of the SMT-EGJ was identified (Fig. 1B). This was achieved using intraoperative endoscopy (IOE), performed by the endoscopic assistant. This technique has been especially useful for the detection of endophytic type SMTs. All endoscopic procedures were performed once endoscopic bowel clamping of the proximal part of jejunum was ensured, so as to prevent unnecessary inflation of the small intestine during surgery. Using laparoscopic monopolar electrocautery and ultrasonic shears, SMT enucleation began with cautious subserosal dissection (Fig. 1C). Once an incision had been made, luminal air-insufflation via IOE was performed by the endoscopist. The SMT was enucleated along the submucosal plane (with caution of bowel perforation) using a laparoscopic electrocautery and coagulating device (Fig. 1D), and laparoscopic sutures were performed on any mucosal injury sustained during the proce- 

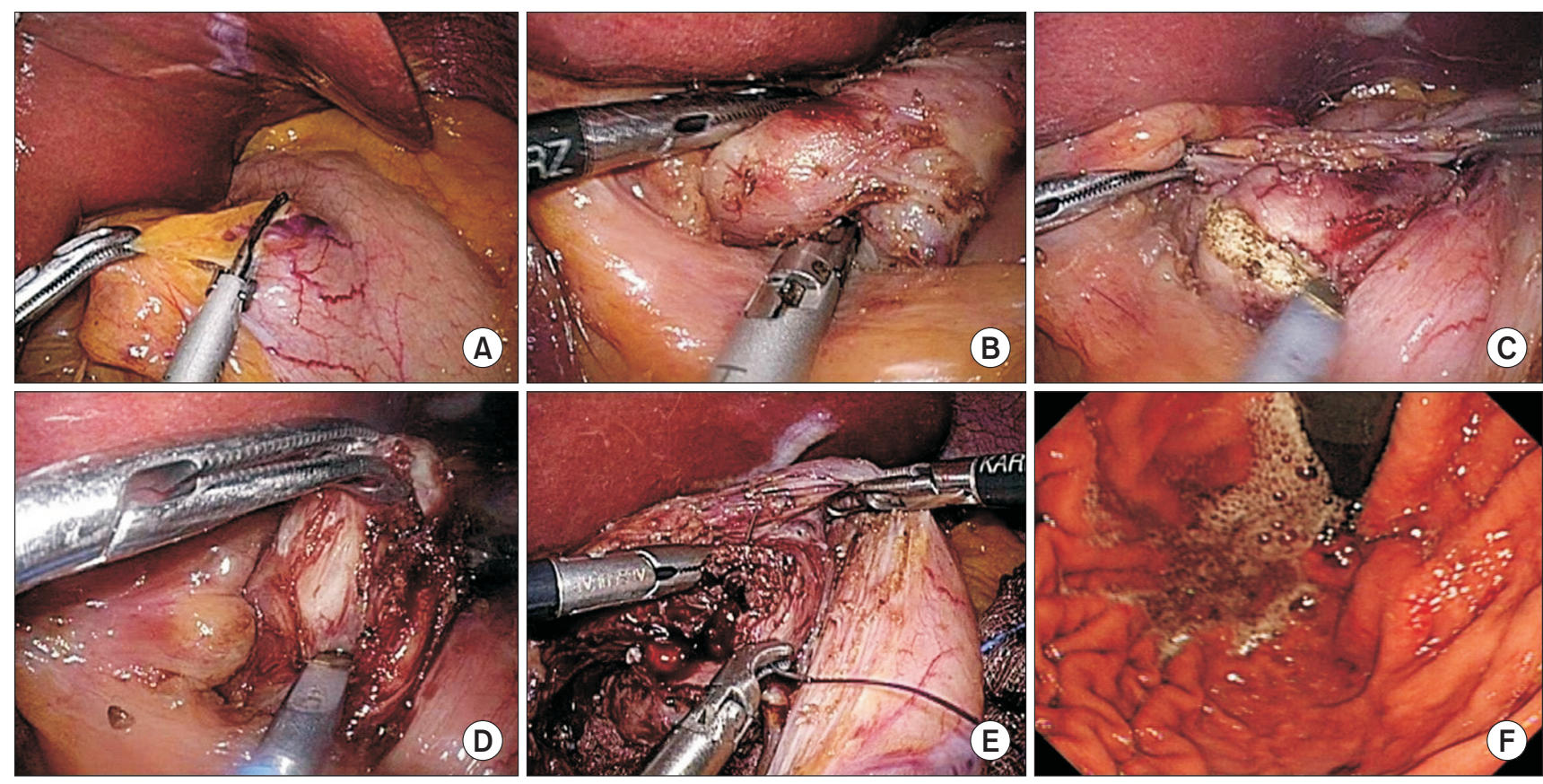

Fig. 1. Steps of Laparoscopic local resection through subserosal dissection with endoscopic air-insufflation (LRSDEA) for gastric submucosal tumors located near the esophagogastric junction (SMT-EGJ); (A) tumor was done by laparoscopic dissection around esophagogastric junction, (B) tumor popped out and identify of submucosal tumor (SMT), (C) subserosal layer was dissected using laparoscopic monopolar electrocautery, (D) SMT was enucleated, (E) seromuscular suture, and (F) intraoperative endoscopic check for resection site.

dure. After tumor enucleation, the seromuscular layer was sealed with an absorbable suture material using an interrupted suture technique for reinforcement (Fig. 1E). The abdominal esophagus was mobilized during dissection, while preserving the left anterior and right posterior vagus nerves. In cases requiring extensive exfoliation of the entire EGJ, fundoplication was performed to prevent reflux after surgery. IOE was performed once more to identify air leakage, bleeding at the resection site, or gastric inlet patency (Fig. 1F). The operative field was irrigated with saline, and a peritoneal drain was placed within the intra-abdominal cavity as a precautionary measure. The specimen was removed using a retrieval plastic bag through the infraumbilical trocar site, and the abdominal wall incisions were closed by layer. These procedures are shown in Fig. 1 and the supplementary video clip.

\section{Postoperative hospital care and follow-up evaluation}

All patients were allowed sips of water 1 day after surgery. An upper gastrointestinal series using water soluble gastrografin was performed on the second postoperative day (or before resuming a diet), and the body fluid levels in the surgical drain, such as amylase, lipase and total bilirubin, were analyzed. On the third postoperative day, a semi-fluid diet was permitted, and on day 4 , a soft blended diet was allowed; here the drain tube was removed unless otherwise specified. Most patients were discharged 6 or 7 days after surgery.

All patients underwent an outpatient follow-up examination 1 month after discharge, where we addressed any symptom reported by the patient, including abdominal discomfort, dyspepsia reflux, or vomiting, in addition to the follow-up tests. A postoperative recurrence test was annually performed using follow-up endoscopy and CT scans.

\section{RESULTS}

The demographic information and clinical characteristics of the patients is summarized in Table 1 . There were 4 male and 5 female patients, with a mean age of 47 years (aged between 37 58 years). The patients were physically classified according to the American Society of Anesthesiologists scoring-system, which indicates a patient's health status before surgery; each patient received a score of 2. Mean body mass index (BMI) was $24.7 \mathrm{~kg} /$ $\mathrm{m}^{2}$ (range: $18.5 \sim 30.1 \mathrm{~kg} / \mathrm{m}^{2}$ ). SMTs were identified at the EGJ of 7 patients, the cardia of another patient, and the lower esophagus of the final patient. Five of the tumors exhibited endophytic growth patterns, whereas the other 4 exhibited both endophytic and exophytic growth. Final pathology revealed 6 leiomyomas, 2 GIST, and 1 gastric duplication. The average size of the resected tumor was $3.5 \mathrm{~cm}$ (range: $1.2 \sim 7.2 \mathrm{~cm}$ ). A clear resection margin was obtained, and R0 resection was confirmed for each of the 
Table 1. Patients' demographics and clinicopathologic characteristics

\begin{tabular}{|c|c|c|c|c|c|c|c|c|}
\hline Case & Gender & Age (yr) & ASA & BMI $\left(\mathrm{kg} / \mathrm{m}^{2}\right)$ & Location & Gross pattern & Pathology & $\begin{array}{c}\text { Tumor } \\
\text { size }(\mathrm{cm})\end{array}$ \\
\hline 1 & Female & 44 & 2 & 20.7 & Cardia & Endophytic & GIST & 1.2 \\
\hline 2 & Male & 37 & 2 & 23.9 & EGJ involved & Both & Gastric duplication & 3.5 \\
\hline 3 & Female & 44 & 2 & 30.1 & EGJ involved & Both & Leiomyoma & 2.2 \\
\hline 4 & Male & 58 & 2 & 25.3 & EGJ involved & Both & GIST & 3.0 \\
\hline 5 & Male & 38 & 2 & 26.1 & EGJ involved & Both & Leiomyoma & 3.0 \\
\hline 6 & Male & 45 & 2 & 23.9 & Lower esophagus & Endophytic & Leiomyoma & 2.0 \\
\hline 7 & Female & 44 & 2 & 18.5 & EGJ involved & Endophytic & Leiomyoma & 4.0 \\
\hline 8 & Female & 56 & 2 & 26.8 & EGJ involved & Endophytic & Leiomyoma & 5.0 \\
\hline 9 & Female & 57 & 2 & 26.8 & EGJ involved & Endophytic & Leiomyoma & 7.2 \\
\hline
\end{tabular}

ASA = American society of anesthesiologist score; BMl = body mass index; EGJ = esophago-gastric junction; GIST = gastrointestinal stromal tumor; Both = endophytic and exophytic type submucosal tumor.

Table 2. Operative and surgical outcomes

\begin{tabular}{lc}
\multicolumn{1}{c}{ Variable } & Value \\
\hline Operation time (minutes, range) & $126.1 \pm 22.0$ (95 260) \\
Intra-operative event & $1^{\dagger}$ \\
Fundoplication & 2 \\
Open conversion & 0 \\
Intraoperative blood loss (ml) & $12 \pm 3.2$ \\
Sips of water start (day) & 1 \\
Diet start (SBD, day) & $3.6 \pm 0.2$ (range, 3 5) \\
Hospital stay (day) & $6.8 \pm 0.6$ (range, 5 11) \\
Postoperative complication & 0 \\
Follow-up period (months) & $15.8 \pm 12.9$ (range, 12 33) \\
Local recurrence & 0
\end{tabular}

Values are presented as mean \pm standard deviation or number. ${ }^{\dagger}$ Mucosal perforation during mass enucleation.

resected tumors.

All procedures of LRSDEA were successfully performed without converting to major gastric resection or open conversion, and no intraoperative complications developed; there were no postoperative mortalities. The mean operation time was $126.1 \pm 22.0$ min (range: 95 260 min), and average blood loss was 12.5 $\pm 3.2 \mathrm{ml}$. While no rupture or spillage of the tumor occurred during surgery, mucosal perforation occurred in 1 case during tumor resection; this was resolved using primary suture. Nissen (case 6) and Toupet (case 9) fundoplication procedures were performed in 2 cases during the LRSDEA to prevent gastro-esophageal reflux.

Postoperative care allowed patients to begin drinking sips of water 1 day after surgery. On average, patients began to consume a soft blended diet 3.6 \pm 0.2 days (range: $3 \sim 5$ days) post-operation. The mean length of postoperative hospital stay was $6.8 \pm 0.6$ days (range: 5 11 days). During the 1-month follow-up, only 1 patient reported experiencing discomfort; the reflux symptoms were successfully treated with a proton pump inhibitor within 2 weeks. There was no evidence of tumor recurrence or metastasis indicated by the CT scan or the EGD during the follow-up period, which averaged 15.8 \pm 12.9 months (range: 12 33 months) (Table 2).

\section{DISCUSSION}

The mainstay of treatment for gastric SMTs is complete surgical resection. Local resection of SMT-EGJ is especially difficult due to its high risk for surgical complications, such as gastric deformity, obstruction after surgery, or stenosis. Minimizing the unnecessary ablation of normal stomach tissue is imperative for the prevention of these complications. In this technical report, we introduced a successful new method for the safe resection of SMT-EGJ. This method, LRSDEA, is particularly advantageous for SMTs displaying an endophytic growth pattern.

Various methods have been proposed for the complete surgical resection of SMT-EGJ, with many laparoscopic techniques being developed as a result. Laparoscopic endoscopic cooperative surgery (LECS) involves seromuscular resection by laparoscopy, using endoscopic dissection between the mucosal and submucosal layers. This makes efficient resection possible independent of tumor location (e.g., at the EGJ or pyloric ring. ${ }^{6,13}$ Tagaya and Choi have reported that intragastric wedge resection, performed by inserting laparoscopic trocars into the stomach, was useful for endoluminal SMT-EGJ resection. ${ }^{11,14}$ Song et al. ${ }^{7}$ and Xu et al. ${ }^{8}$ have suggested a transgastric approach as an alternative method 
for treating endophytic tumors. The technique, which has a safe resection margin, allows identification of the tumor location using laparoscopy, while preserving the normal gastric wall. Kwon et al. ${ }^{10}$ have described endoscopic and laparoscopic full-thickness resection, with laparoscopic two-layer suturing for SMT-EGJ. Suturing of the seromuscular layer has the advantage of preserving the mucosal layer, as well as maintaining the diameter. However, these techniques pose certain limitations; while endoscopic submucosal dissection requires a certain level of skill, there is the additional risk of intraperitoneal tumor dissemination, as well as gastric juice contamination of the abdominal cavity due to opening of the gastric wall. Furthermore, unnecessary gastric injury to normal tissue could increase the likelihood of gastric volume reduction, functional deterioration, stenosis, and leakage.

Through the application of LRSDEA, these disadvantages could be overcome by enabling more precise target detection by endoscopy, and avoiding both normal gastric wall injury and gastrectomy. Results indicate that there were no intra- or postoperative surgical complications, and follow-up data indicated that most patients did not experience symptoms of reflux, abdominal discomfort, or difficulty swallowing.

IOE is considered an important tool in surgical planning, as our technique, LRSDEA, makes active use of the method. Additionally, IOE has proven a useful adjunct to gastric surgery. Precise endoscopic localization of the tumor during surgery has helped to maintain the correct margin of resection throughout the laparoscopic gastrectomy. ${ }^{15}$ Moreover, IOE offers an additional advantage, by combining this leak-check with a visual inspection of the mucosal surfaces, and the anastomosis itself. This provides an opportunity to diagnose and treat staple-line hemorrhage, as well as to verify the adequacy of the resection. ${ }^{16}$ This is easily accomplished via endoscopy to overcome the difficulty of detecting the tumor from the serosal side. Endoscopic air-insufflation during subserosal dissection has the advantage of causing a visual separation the normal gastric tissue and the tumor, allowing for easy identification in the laparoscopic field of view. Finally, using IOE, we were able to evaluate postresection complications such as passage disturbance, stenosis, stricture, bleeding, or air leakage of the EGJ. It thus serves as an effective mode for the prevention of gastric inlet stenosis.

A fundoplication was performed on the 2 patients displaying a possible risk for reflux either due to damage of the antireflux mechanism (case 9) or uncontrolled gastroesophageal reflux symptoms (case 6). Two patients were taking antireflux drugs such as proton pump inhibitor because they complained of severe symptoms of reflux on preoperative surgery. During LRSDEA, excessive exfoliation around EGJ is required for the removal of the tumor, a process which could lead to the destruction of the intrinsic musculature of the distal esophagus, the sling fibers of the gastric cardia, and the crura of the diaphragm that comprise the lower esophageal sphincter. Each of the aforementioned complications have the potential to cause serious reflux after surgery; thus, for the safe application of LRSDEA, additional antireflux surgery such as fundoplication may be required. This would depend on both the patient's preoperative reflux symptoms, as well as the degree of lower esophageal sphincter damage. Two patients were taking antireflux drugs such as proton pump inhibitor because they complained of severe symptoms of reflux on preoperative surgery. And then, all patients did not complain of reflux symptoms or needed medication after surgery.

The most important objective during resectable primary GIST excision is maintaining a free resection margin without tumor rupture. ${ }^{17}$ The disadvantage of LRSDEA is that the tumor is constantly handled during the enucleation process, posing a risk for either injury or rupture of the tumor. This procedure should therefore not be performed if preoperative CT scans, EGD, or EUS tests suggest malignant GISTs larger than $5 \mathrm{~cm}$; accurate preoperative examinations and interpretation by radiologic and endoscopic experts are therefore essential. In our report, no tumor damage was sustained during surgery. Our final pathological analyses identified 7 benign tumors such as leiomyomas or duplication cysts, and 2 GISTs. Based on NIH criteria, the GISTs were identified as very low-risk, as they were smaller than $3 \mathrm{~cm}$ and exhibited no more than 5 mitoses/50 high-power fields. ${ }^{18}$

There are several recommendations for the safe performance of LRSDEA; whenever a laparoscopic subtumoral dissection is performed, meticulous handling of the SMT is prerequisite for the prevention of tumor damage. In addition, it is recommended that rather than holding the tumor directly, the soft tissue surrounding the tumor should be gently grasped. Avoiding damage to the tumor or normal mucosa by electrocautery or energy device is another important technical tip. Confirmation of tumor after subserosal layer dissection and subsequent endoscopic airinsufflation after detachment of surrounding muscle layer could enable easy enucleation, as the tumor visibly protrudes. Endoscopic air-insufflation after tying up the gastric body with surgical nylon tape may additionally be beneficial for securing vision around the EGJ, as this could prevent distal stomach dilatation. It should be noted that endoscopic air injection should not be excessively used to prevent mucosal layer injury; if mucosal injury does occur during the tumor enucleation process, the surgeon should detect and repair immediately. In our report, 1 injury was sustained and a subsequent a repair was performed.

The most important advantage of LRSDEA for SMT-EGJ is the maintenance of a normal EGJ without major gastric resection, such as total or proximal gastrectomy. Many gastric surgeons are hesitant toward the excision of SMT-EGJ due to the possibility of EGJ malfunction. This method would henceforth serve as a strategic tool, especially for the treatment SMT-EGJ displaying endophytic growth patterns. Nevertheless, several limitations 
should be considered. First, careful dissection around the tumor is a complex procedure that, in addition to being time-consuming, requires both experience and specialized laparoscopic skill. Second, the procedure cannot be performed on large, irregular tumors with a high probability for malignancy.

In summary, LRSDEA appears to be a useful and safe procedure for the resection of SMT-EGJ with low malignancy, and an endophytic growth pattern. Furthermore, it may be used as the main surgical technique with the added advantage of avoiding major gastric surgeries such as a proximal or total gastrectomy.

\section{ORCID}

In-Kyeong Kim, https://orcid.org/0000-0003-0765-5985

Ji-Ho Park, https://orcid.org/0000-0002-2751-7320

Young-Joon Lee, https://orcid.org/0000-0002-1735-3385

Sang-Ho Jeong, https://orcid.org/0000-0001-9786-5710

Tae Han Kim, https://orcid.org/0000-0002-5012-7208

Dong-Hwan Kim, https://orcid.org/0000-0002-2151-0048

Han-Gil Kim, https://orcid.org/0000-0002-6394-942X

Jin-Kyu Cho, https://orcid.org/0000-0002-6877-7620

Jae-Myung Kim, https://orcid.org/0000-0002-9012-8176

Seung-Jin Kwag, https://orcid.org/0000-0002-9267-9158

Ju-Yeon Kim, https://orcid.org/0000-0002-5846-7522

Chi-Young Jeong, https://orcid.org/0000-0003-4121-6695

Young-tae Ju, https://orcid.org/0000-0002-1785-8500

Eun-Jung Jung, https://orcid.org/0000-0001-8413-613X

Soon-Chan Hong, https://orcid.org/0000-0003-4499-8741

\section{AUTHORS' CONTRIBUTIONS}

Conceptualization: Young-Joon Lee, Ji-Ho Park, and In-Kyeong Kim. Formal analysis: Sang-Ho Jeong and Tae Han Kim. Methodology: Han-Kil Kim, Jin-Kyu Cho, and Seung-Jin Kwag. Writing-original draft: In-Kyeong Kim, Dong-Hwan Kim, and Ji-Ho Park. Writing-review and editing: Jae-Myung Kim, Ju-Yeon Kim, Chi-Young Jeong, Yoiung-tae Ju, Eun-Jung Jung, and Soon-Chan Hong.

\section{CONFLICT OF INTEREST}

None.

\section{FUNDING}

None.

\section{ACKNOWLEDGMENTS}

None.

\section{REFERENCES}

1) Kang YK, Kim KM, Sohn T, et al. Clinical practice guideline for accurate diagnosis and effective treatment of gastrointestinal stromal tumor in Korea. J Korean Med Sci 2010;25:1543-1552.

2) Choi YB, Oh ST. Laparoscopy in the management of gastric submucosal tumors. Surg Endosc 2000;14:741-745.

3) Matthews BD, Walsh RM, Kercher KW, et al. Laparoscopic vs open resection of gastric stromal tumors. Surg Endosc 2002;16:803-807.

4) Hyung WJ, Lim JS, Cheong JH, Kim J, Choi SH, Noh SH. Laparoscopic resection of a huge intraluminal gastric submucosal tumor located in the anterior wall: eversion method. J Surg Oncol 2005;89:9598.

5) Hwang SH, Park DJ, Kim YH, et al. Laparoscopic surgery for submucosal tumors located at the esophagogastric junction and the prepylorus. Surg Endosc 2009;23:1980-1987.

6) Hoteya $S$, Haruta $S$, Shinohara $H$, et al. Feasibility and safety of laparoscopic and endoscopic cooperative surgery for gastric submucosal tumors, including esophagogastric junction tumors. Dig Endosc 2014;26:538-544.

7) Song KY, Kim SN, Park CH. Tailored-approach of laparoscopic wedge resection for treatment of submucosal tumor near the esophagogastric junction. Surg Endosc 2007;21:2272-2276.

8) $\mathrm{Xu} \mathrm{X}$, Chen $\mathrm{K}$, Zhou W, et al. Laparoscopic transgastric resection of gastric submucosal tumors located near the esophagogastric junction. J Gastrointest Surg 2013;17:1570-1575.

9) Schmidt A, Bauder M, Riecken B, von Renteln D, Muehleisen H, Caca K. Endoscopic full-thickness resection of gastric subepithelial tumors: a single-center series. Endoscopy 2015;47:154-158.

10) Kwon OK, Yu W. Endoscopic and Laparoscopic Full-Thickness Resection of Endophytic Gastric Submucosal Tumors Very Close to the Esophagogastric Junction. J Gastric Cancer 2015;15:278-285.

11) Choi CI, Lee SH, Hwang SH, et al. Single-incision intragastric resection for upper and mid gastric submucosal tumors: a case-series study. Ann Surg Treat Res 2014;87:304-310.

12) George D Demetri MvM, Cristina R Antonescu, Ronald P DeMatteo, Kristen N Ganjoo, Robert G Maki, Peter W T Pisters, Chandrajit P Raut, Richard F Riedel, Scott Schuetze, Hema M Sundar, Jonathan C Trent, Jeffrey D Wayne NCCN Task Force report : update on the management of patients with gastrointestinal stromal tumors. J Natl Compr Canc Netw 2010;8:S1-41; quiz S42-44.

13) Hiki N, Yamamoto Y, Fukunaga T, et al. Laparoscopic and endoscopic cooperative surgery for gastrointestinal stromal tumor dissection. Surg Endosc 2008;22:1729-1735.

14) Tagaya N, Mikami H, Kogure H, Kubota K, Hosoya Y, Nagai H. Laparoscopic intragastric stapled resection of gastric submucosal tumors located near the esophagogastric junction. Surg Endosc 2002;16:177179.

15) Park DJ, Lee HJ, Kim SG, et al. Intraoperative gastroscopy for gastric surgery. Surg Endosc 2005;19:1358-1361. 
16) Park JH, Jeong SH, Lee YJ, et al. Safety and efficacy of postanastomotic intraoperative endoscopy to avoid early anastomotic complications during gastrectomy for gastric cancer. Surg Endosc 2020;34:5312-5319.

17) Nishida $T$, Hirota $S$, Yanagisawa A, et al. Clinical practice guidelines for gastrointestinal stromal tumor (GIST) in Japan: English version. Int J Clin Oncol 2008;13:416-430.

18) Fletcher CD, Berman JJ, Corless $C$, et al. Diagnosis of gastrointestinal stromal tumors: a consensus approach. Int J Surg Pathol 2002;10:8189. 